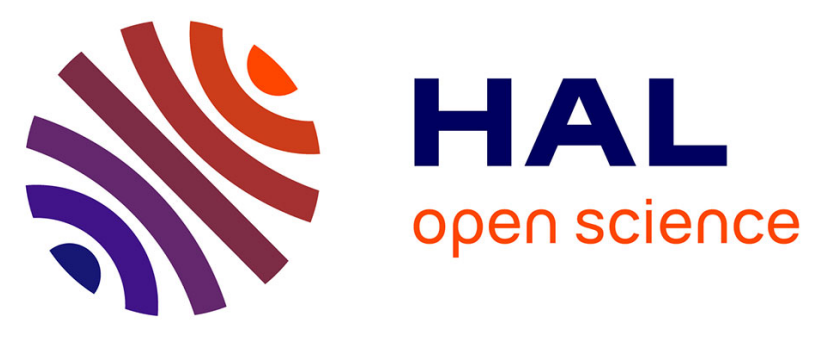

\title{
Understanding the Impact of User Behaviours and Scheduling Parameters on the Effectiveness of a Terminal Appointment System Using Discrete Event Simulation
}

Mihai Neagoe, Hans-Henrik Hvolby, Mohammad Sadegh Taskhiri, Paul Turner

\section{- To cite this version:}

Mihai Neagoe, Hans-Henrik Hvolby, Mohammad Sadegh Taskhiri, Paul Turner. Understanding the Impact of User Behaviours and Scheduling Parameters on the Effectiveness of a Terminal Appointment System Using Discrete Event Simulation. IFIP International Conference on Advances in Production Management Systems (APMS), Sep 2019, Austin, TX, United States. pp.27-34, 10.1007/978-3-03029996-5_3. hal-02460518

\section{HAL Id: hal-02460518 \\ https://hal.inria.fr/hal-02460518}

Submitted on 30 Jan 2020

HAL is a multi-disciplinary open access archive for the deposit and dissemination of scientific research documents, whether they are published or not. The documents may come from teaching and research institutions in France or abroad, or from public or private research centers.
L'archive ouverte pluridisciplinaire HAL, est destinée au dépôt et à la diffusion de documents scientifiques de niveau recherche, publiés ou non, émanant des établissements d'enseignement et de recherche français ou étrangers, des laboratoires publics ou privés.

\section{(ㄷ)(i)}

Distributed under a Creative Commons Attribution| 4.0 International License 


\title{
Understanding the Impact of User Behaviours and Scheduling Parameters on the Effectiveness of a Terminal Appointment System Using Discrete Event Simulation
}

\author{
Mihai Neagoe 1(凶)[0000-0003-4362-8132], Hans-Henrik Hvolby ${ }^{2,3[0000-0002-5574-5216]}$ Moham- \\ mad Sadegh Taskhiri ${ }^{10000-0002-9871-361 X]}$, and Paul Turner ${ }^{10000-0003-4504-2338]}$ \\ ${ }^{1}$ ARC Centre for Forest Value, Discipline of ICT, College of Sciences and Engineering, Uni- \\ versity of Tasmania, Hobart, Australia \\ $\triangle$ Mihai.Neagoe@utas.edu.au \\ ${ }^{2}$ Centre for Logistics, Department of Materials \& Production, Aalborg University, Aalborg, \\ Denmark \\ ${ }^{3}$ Department of Mechanical and industrial Engineering, Norwegian University of Science and \\ Technology, Trondheim, Norway
}

\begin{abstract}
This research improves understanding of the impact of specific types of truck driver behaviour and temporal scheduling on the effectiveness of a terminal appointment system. A discrete event simulation model of a bulk cargo marine terminal is developed to analyse parameters related to driver behaviour (punctuality and proportion of planned appointments) and temporal scheduling (appointments per time window and time window spacing) on truck flows and turnaround times at the terminal. The model is based on an Australian wood chip export marine terminal currently experiencing significant truck congestion. The terminal operator and stakeholders have expressed interest in the implementation of an appointment system to address this issue. The modelling presented in this research was used to inform their investigation into developing an appointment system solution.

Simulation results indicate that the proportion of planned appointments, used as a proxy for the appointment system use, has a significant impact on truck turnaround times. Greater truck arrival punctuality only marginally improves truck turnaround times. Interestingly most optimization approaches continue to focus on improving punctuality through service rules or financial penalties in order to achieve optimal turnaround times. However, the additional cost in terms of complexity or assumptions for optimal solutions against non-optimal approaches are rarely weighed in terms of dividends of the marginal improvements generated. By involving terminal users (drivers and transporters) in the design of an appointment system and its scheduling parameters, terminal operators can significantly improve appointment system use and effectiveness by increasing the probability of positive users' behaviours.
\end{abstract}

Keywords: Transport management, supply chain collaboration, user requirements, congestion management. 


\section{Introduction}

Terminal appointment systems are one of the most effective methods to manage congestion and communicate with multiple users at marine terminals. The system's performance in terms of truck turnaround times and equipment use efficiency can be affected by the systems' parameters and by the behaviours of the terminal users. System parameters can include the lead time for selecting an appointment time, appointment window length [1], number of appointments per time-window [2, 3], appointment spacing, and truck servicing rules [4]. User behaviours can be modelled by the probability that drivers miss appointments, arrive un-appointed [3], or their arrival punctuality [4, 5]. The system parameters are often determined by optimization approaches, either linear programming [6], queuing theory [7] or through simulation [8]. Some user behaviours are seen as disruptions to the optimal system solution that must be dealt with. Authors recommend the use of penalty systems to enforce compliance with the appointment system schedules [5] while others introduce more complex truck service rules to maintain a high level of system efficiency [4]. Although enforcement and system rules approaches have merit in moderating users' behaviours they suffer from a series of shortcomings:

- The information required for complex system rules may be difficult to collect in realtime, making implementations in real-life scenarios challenging;

- Neither approach (enforcement nor system rules) is based on understanding of the underlying causes behind the users' behaviours. This can lead to unexpected outcomes such as system misuse [9].

- Users' involvement in decisions regarding the system's parameters and functionality is typically limited [10]. The potential consequences of the lack of involvement in decision making is explained by Ackoff [11]: "In problems, the solution to which involve the reactions of others, their participation in the problem-solving process is the best protection against unexpected responses [...] A failure to consult others who have a stake in our decision is often seen as an act of aggression".

This research therefore introduces a discrete event simulation model of a bulk cargo marine terminal is developed to analyse parameters related to driver behaviour (punctuality and proportion of planned appointments) and temporal scheduling (appointments per time window and time window spacing) on truck flows and turnaround times at the terminal. The modelling results are then used to support the involvement of terminal users prior to the implementation of an appointment system to understand their requirements, consequences on the users' behaviours and the consequences of the users' behaviours on the effectiveness of the system.

\section{The Wood Chip Export Terminal Field Site}

The marine terminal on which the modelling work is based is an Australian wood chip export facility. Wood chips are processed from logs in facilities located in close proximity of the terminal, and delivered to the terminal. Wood chips are stored at the terminal and subsequently loaded on dry bulk cargo ships belonging to international pulp 
and paper producers. The terminal receives approximately 1.6 million tons of wood chips and handles roughly 52,000 truck deliveries per year. The terminal's customers outsourced the transportation task between their production sites and the terminal to a stable base of transport contractors. This setup creates a small and relatively closed transport system. At the terminal, trucks are first weighed at a weigh-bridge. Next trucks drive to an on-dock staging area where they wait for one of two unloading ramps to become available. Once a ramp is available, trucks are unloaded, then drive to a weigh-bridge to be weighed once more. The terminal and supply chain setup have been explored in greater detail in previous work [12].

The terminal was experiencing truck significant congestion. The consequences of congestion ranged from increased costs for transporters, increased supply chain uncertainty for terminal customers, and additional staff and maintenance scheduling for the terminal operator. The stakeholders were considering a range of potential options to mitigate congestion and expressed interest in an appointment system to manage truck flows at the terminal. The researchers developed a simulation model to further understanding regarding the potential consequences of different system parameters and behaviours can have on truck waiting and turnaround times and ultimately inform the stakeholders' investigation into the development of an appointment system solution.

\section{$3 \quad$ Data Collection and Simulation Model}

Data on truck arrivals were collected from the terminal weigh-bridge database and geolocation data from GPS units mounted on several trucks. These data were supplemented by on-site observations by the research team and discussions with stakeholders. The GPS data were used in conjunction with geo-fences setup around terminal infrastructure that quantified the duration of truck visits at every stage. The truck arrival frequency and geo-fence visit durations data were analysed with Arena Input Analyzer to generate distributions. Approximately 7 months of truck arrival data and 3 months of geo-location data were used. The fitted distributions formed the input for the discrete-event simulation model.

The simulation model, presented in Table 1, comprises of two stages: the truck arrival generator and the truck processing. The lack of complexity in the model's design is purposeful, as the model and results primarily served as a discussion point with the terminal's and its users' staff which had a diverse demographic and socio-economic backgrounds. The use of a relatively simplistic model aimed to improve its accessibility to a broader audience. The model is implemented in Python programming language.

In the first stage, the truck arrivals are generated. The planned arrivals parameter indicates the percentage of appointed arrivals and walk-ins. The arrival time of the truck is then calculated based on whether or not the respective truck has an appointment. A time-window spacing coefficient is applied on each one-hour interval (e.g. a 6-minute spacing means that within a 60-minute interval, the first 6 minutes do not have any appointments). The truck arrival list is then sorted in ascending order of arrival times and fed to a generator function which creates truck objects with payload, capacity, product characteristics. 
Table 1. Simulation model stages and steps

\begin{tabular}{|c|c|}
\hline Step & Stage 1 - Truck Arrival Generator \\
\hline 1.1 & Random choice of appointed/un-appointed arrival; \\
\hline $1.2 \mathrm{~A}$ & $\begin{array}{l}\text { If un-appointed arrival: arrival time }=\text { previous truck arrival time }+ \\
\text { inter-arrival time (gamma, } \mathrm{k}=1.49, \theta=6.97)\end{array}$ \\
\hline $1.2 \mathrm{~B}$ & $\begin{array}{l}\text { Else appointed arrival: arrival time }=\text { previous app. time }+ \text { spacing }+ \\
\text { appointment interval }+ \text { punctuality; }\end{array}$ \\
\hline 1.3 & Sort truck list based on truck arrival times; \\
\hline 1.4 & Generate truck object (payload, capacity, product and arrival times); \\
\hline Step & Stage 2 - Truck Processing \\
\hline 2.1 & Next truck object from arrival list; \\
\hline 2.2 & Weigh-in at Weigh-bridge ( $1 \mathrm{~min})$; \\
\hline 2.3 & Drive to Unloading area $(1 \mathrm{~min})$ \\
\hline $2.4 \mathrm{~A}$ & $\begin{array}{l}\text { If any unloading ramp free and available: Unloading } \\
\text { lognormal }(\mathrm{m}=5.16, \mathrm{~s}=3.97)\end{array}$ \\
\hline $2.4 \mathrm{~B}$ & Else no unloading ramp free and available: Accrue waiting time; \\
\hline 2.5 & Drive to Weigh-bridge - $2 \mathrm{~min}$; \\
\hline $2.6 \mathrm{~A}$ & If weigh-bridge free: Weighing - normal $(\mu=3.46, \sigma=1.68)$; \\
\hline $2.6 \mathrm{~B}$ & Else weigh-bridge not free: Accrue waiting time; \\
\hline 2.7 & Calculate total service and waiting times for truck; \\
\hline
\end{tabular}

In the second model stage, the truck objects are then processed by the terminal object. The two-stage approach is required as trucks are served on a first-come, firstserved basis, irrespective of their appointment time. While other priority rules are considered in the literature [4], The first-come, first served approach was considered in this model for its lower level of complexity. The second stage is largely based on modelling presented in previous work [13]. The weigh-out and unloading ramp service times are stochastically determined from distributions fitted from GPS data. The weigh-in in stage is held constant at 1 minute/truck. the drive times from the weigh-bridge and unloading ramp and back are held constant at 1 and 2 minutes respectively. The total truck processing times including the waiting are the summarized and output.

The second simulation stage follows closely the unloading process observed by the research team at the terminal and described in Section 2. The simulation model and its logic have been presented and discussed with terminal staff to improve the accuracy and validity of the representation.

\section{Scenario Analysis: System Parameters and User Behaviour}

The four factors included in the scenario analysis were driver behaviours (punctuality, missed and unplanned appointments) and system parameters (appointments per time window and appointment buffers). These factors were adapted from the appointment systems literature. The factors included are:

- Number of appointments per time window. Two values were included for each one-hour time window, 6 (low frequency) and 8 appointments (high frequency). In 
the cases where all appointments were unplanned, an inter-arrival time distribution that would provide similar arrival frequency was used.

- Time window spacing. Each time window contains the same number of appointments but has a starting buffer period. The three values included were 0-, 6-, and 12minutes/time window.

- Planned/Unplanned arrivals. The proportion of planned and unplanned arrivals was varied in 25\% increments between $0 \%$ (all un-appointed arrivals) and $100 \%$ (all appointed arrivals).

- Arrival punctuality. Punctuality was modelled by adding a stochastic component to each appointed arrival time. Three normal distributions were used to simulate truck arrival punctuality, similar to the approach presented in [5]: (1) High: $95 \%$ of arrivals are within $+/-5$ min. from appointment time $\{\mathrm{N}(0,2.5)\}$, (2) Medium: $68 \%$ of arrivals are within $+/-5$ min. from appointment time $\{\mathrm{N}(0,5)\}$, (3) Low: $38 \%$ of arrivals are within $+/-5$ min. from the appointment time $\{\mathrm{N}(0,10)\}$,

The scenario analysis included combinations of the 4 factors and resulted in 74 scenarios. Each scenario was run 20 times and each iteration simulated a year of operations.

\section{$4 \quad$ Modelling Results}

The results of the simulation model in terms of average truck turnaround time for the scenarios tested are presented in Fig. 1. The scenario where an average of 6 trucks per hour arrive uncoordinated resembles the situation empirically observed at the terminal (yellow diamond symbol). The modelled turnaround time in this scenario was approximately 23.5 minutes per truck. This turnaround time figure is similar to the empirically observed average turnaround time. The turnaround time in this scenario includes an average waiting time of 6 minutes per truck, the majority of which accrued waiting for an unloading ramp to become available. The sequential nature of the processes at the terminal means that reductions in turnaround time arise primarily from a reduction in waiting times. Since the terminal's infrastructure is fixed, most benefits accrued from the reduction in waiting and turnaround times directly benefit terminal users.

The change from a low to high frequency truck arrivals generates a $20 \%$ increase in throughput, from 1.6 to 2 million tons. The change in truck arrival frequency is represented in Fig. 1 by the change in symbol colours, from yellow to blue. This increase is also met with a doubling of truck turnaround times, most likely due to throughput getting closer to the terminal's maximum physical capacity. In the higher arrival frequency scenarios, an improvement in turnaround times of 25 to $30 \%$, is generated if at least $25 \%$ of arrivals are scheduled. The turnaround time improvement gradually decreases as a higher percentage of trucks arrive appointed. When all trucks arrive with appointments, the expected turnaround time improvement is between 37 and $46 \%$ compared to the scenario where all appointments are unplanned, depending on arrival punctuality. In the low arrival frequency scenarios, the marginal improvement with each increment of planned appointment proportion remains relatively constant, between 3 and 5\%, depending on the arrival punctuality. 


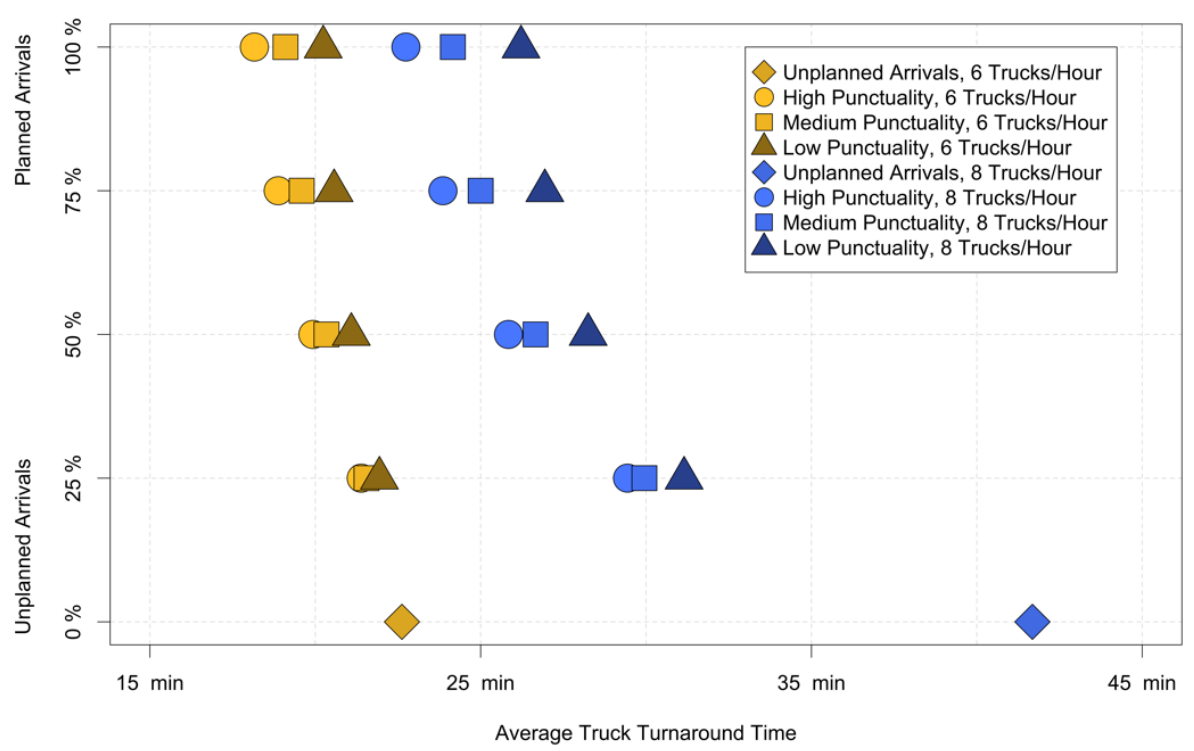

Fig. 1. Simulation model scenario analysis results in terms of average truck turnaround times, truck arrival punctuality, and percentage of planned arrivals

The impact of low arrival punctuality increases with the proportion of appointed truck arrivals. Punctuality is represented in Fig. 1 by the change of symbol type and darkening colour tones. Low punctuality halves the effectiveness of the appointment system compared to high punctuality in virtually all low arrival frequency scenarios. In high arrival frequency scenarios, the impact of low punctuality is less considerable, between 16-20\% compared to high punctuality. Time window spacing (not depicted in Fig. 1) has limited impact on truck turnaround time averages. The introduction of 6- or 12 -minute spacing between each time window increases turnaround times by $2 \%$ compared to no spacing. The next section discusses the simulation results in the context of the extant literature and of the potential applications.

\section{$5 \quad$ Discussion and Future Research}

Simulation results indicate that truck turnaround times increase non-linearly with throughput which corroborates literature findings [12,14]. Particularly in the high frequency arrival scenarios, a small proportion of known, even less punctual, arrivals, can have a significant impact on turnaround times, similar to [15]. Finally, the impact of arrival punctuality, while an important influencing factor for turnaround times, was not the most important determining factor in turnaround times.

Modelling results revealed that the factor with the most influence on turnaround times was the use of the system. If the potential achievable reduction in turnaround times through the use of the terminal appointment system is not fully appreciated by its users, it is likely that the system use will not be as high as expected. This situation can create 
a vicious circle in which low use reduces the impact of the appointment system on turnaround times therefore leading to lower system use. Therefore, it is paramount to involve users in the design of appointment systems and particularly in determining its operating parameters to encourage system use. While not directly considered in this paper, it is important to acknowledge that the appointment system usability and adoption, among other factors can also influence the system use. Design for usability should also be central in design workshops.

The impact of arrival punctuality was important but considerably less than that of appointment system use. Much of the extant literature focuses on optimizing costs [1, 14] in which case, low truck arrival punctuality can increase terminal and user costs and consequently, the optimal solution. By focusing solely on optimal solution, however, its potential cost in complexity or assumptions compared to near-optimal solutions is rarely weighed against the additional benefits an optimal solution can generate. To ensure compliance from this perspective, variations in service rules [4] or financial penalties have been proposed. However, the literature rarely considers whether the impact of disruptions caused by driver behaviours are sufficiently significant to warrant the introduction of complex service rules and financial penalties variations while risking to reduce the appointment system use.

Future research aims to use the findings from the scenario analysis in conducting participatory design workshops with the terminal operator and its users. The collaboration with the terminal operator is part of an ongoing multiple case-study investigation on mechanisms and technologies to address maritime terminal land-side congestion. The simulation and participatory design approach will be extended to the other case studies to seek replicating the results or identifying differentiating factors.

\section{Conclusion}

This research introduced a discrete event simulation model of a bulk cargo marine terminal is developed to analyse parameters related to driver behaviour (punctuality and proportion of planned appointments) and temporal scheduling (appointments per time window and time window spacing) on truck flows and turnaround times at the terminal.

Modelling findings highlight the importance of involving terminal users in the design of the appointment system and its parameters to ensure its use and consequently its effectiveness in reducing truck turnaround times. Arrival punctuality appears to have less impact than the appointment system use. However, the lack of punctuality is typically financially penalized which tends to cause tensions between terminals and transporters. Shifting focus from enforcing punctuality towards ensuring use of system may assist have a more positive impact on turnaround times.

In the context of this research it is important to acknowledge some of its limitations. The model scope is limited to the terminal gate and unloading operations as insufficient data were available to generate a model of the entire chain. Bulk cargo marine terminal operations are typically less complex than those observed in container terminals, on which the majority of the terminal appointment systems literature is based. It is likely 
that insights generate in this research can be, at least in part, transferrable to other bulkcargo and container marine terminals.

This research is part of an ongoing project undertaken in Australia funded by the Australian Research Council through the Industrial Transformation Research Program.

\section{References}

1. Chen, G., Govindan, K., Yang, Z.: Managing truck arrivals with time windows to alleviate gate congestion at container terminals. Int. J. Prod. Econ. 141, 179-188 (2013).

2. Torkjazi, M., Huynh, N., Shiri, S.: Truck appointment systems considering impact to drayage truck tours. Transp. Res. Part E Logist. Transp. Rev. 116, 208-228 (2018).

3. Huynh, N., Walton, C.M.: Robust Scheduling of Truck Arrivals at Marine Container Terminals. J. Transp. Eng. 134, 347-353 (2008).

4. Li, N., Chen, G., Govindan, K., Jin, Z.: Disruption management for truck appointment system at a container terminal: A green initiative. Transp. Res. Part D Transp. Environ. 61, 261-273 (2018).

5. Ramírez-Nafarrate, A., González-Ramírez, R.G., Smith, N.R., Guerra-Olivares, R., Voß, S.: Impact on yard efficiency of a truck appointment system for a port terminal. Ann. Oper. Res. 258, 195-216 (2017).

6. Chen, G., Jiang, L.: Managing customer arrivals with time windows: a case of truck arrivals at a congested container terminal. Ann. Oper. Res. 244, 349-365 (2016).

7. Guan, C., Liu, R.: Modeling Gate Congestion of Marine Container Terminals, Truck Waiting Cost, and Optimization. Transp. Res. Rec. J. Transp. Res. Board. 2100, 58-67 (2009).

8. Huynh, N., Walton, M.: Improving efficiency of drayage operations at seaport container terminals through the use of an appointment system. In: Handbook of terminal planning. pp. 323-344. Springer, New York, NY (2011).

9. Morais, P., Lord, E.: Terminal Appointment System Study. Transp. Res. Board. 1, 123 (2006).

10. Huynh, N., Smith, D., Harder, F.: Truck Appointment Systems. Transp. Res. Rec. J. Transp. Res. Board. 2548, 1-9 (2016).

11. Ackoff, R.: The art of problem solving. John Wiley \& Sons, New York, Chichester, Brisbane, Toronto, Singapore (1978).

12. Neagoe, M., Taskhiri, M.S., Nguyen, H.-O., Hvolby, H.-H., Turner, P.: Exploring congestion impact beyond the bulk cargo terminal gate. In: Logistics 4.0 and Sustainable Supply Chain Management, Proceedings of HICL 2018. pp. 63-82 (2018).

13. Neagoe, M., Taskhiri, M.S., Nguyen, H.-O., Turner, P.: Exploring the Role of Information Systems in Mitigating Gate Congestion Using Simulation: Theory and Practice at a Bulk Export Terminal Gate. In: IFIP International Conference on Advances in Production Management Systems. pp. 367-374. Springer International Publishing (2018).

14. Guan, C., Liu, R.: Container terminal gate appointment system optimization. Marit. Econ. Logist. 11, 378-398 (2009).

15. Chen, G., Govindan, K., Yang, Z.Z., Choi, T.M., Jiang, L.: Terminal appointment system design by non-stationary $\mathrm{M}(\mathrm{t}) / \mathrm{E} \mathrm{k} / \mathrm{c}(\mathrm{t})$ queueing model and genetic algorithm. Int. J. Prod. Econ. 146, 694-703 (2013). 